\title{
Fabrication of Sponges from Amnion Hydrogel to Apply as Wound Dressing
}

\author{
Nghia Thi Hieu Phan ${ }^{1,2,3}$, My Thi Ngoc Nguyen ${ }^{1,2,3}$ and Ha Le Bao Tran ${ }^{1,2,3, *}$ \\ ${ }^{I}$ Laboratory of Tissue Engineering and Biomedical Materials, University of Science, \\ Ho Chi Minh City, Vietnam \\ ${ }^{2}$ Department of Physiology and Animal Biotechnology, Faculty of Biology and Biotechnology, \\ University of Science, Ho Chi Minh City, Vietnam \\ ${ }^{3}$ Vietnam National University, Ho Chi Minh City, Vietnam
}

('Corresponding author's e-mail: tlbha@hcmus.edu.vn)

Received: 17 March 2021, Revised: 17 May 2021, Accepted: 27 May 2021

\begin{abstract}
A burn is a type of injury to the skin or other tissues. It can give rise to skin defects or even death. In this study, sponges derived from amnion hydrogel were generated to apply as a wound dressing. The sponges were created by combining crosslinking and freeze-drying methods. There were three types of the obtained sponges: MGA-0 (only washed with PBS), MGA-1 (washed in glycine $1 \%$ for one day) and MGA-2 (washed in glycine $1 \%$ for two days). These sponges were evaluated by scanning electron microscope (SEM), mechanical test, swelling, and cytotoxicity. The tensile strength of the sponges was about $1.8 \mathrm{MPa}$, and the absorption increased during $24 \mathrm{~h}$. The relative growth rates (\%RGR) of MGA- 0 , MGA-1, and MGA-2 were 88.8, 58.2 and $67.2 \%$, respectively. The obtained results suggested that the MGA-0 sponge has potential for wound dressing application.
\end{abstract}

Keywords: Amnion hydrogel, Wound dressing, Freeze-drying, Glutaraldehyde, Sponge

\section{Introduction}

According to the World Health Organization (WHO) estimates, about 180000 deaths from burns are reported from the low and middle-income countries each year [1]. A burn is a significant medical problem in the world. Burn wound causes skin structure and function injuries, seriously impairing the body temperature, fluid balance, and physical obstacle to harmful environmental effects $[2,3]$.

For burn treatment, autologous skin grafting is considered the gold standard. However, it still has some limitations when the patients have a large burn or the treatment time is quite long [3,4]. A current trend focuses on generating a membrane that can cover the burn wound to prevent outside infection, stimulate proliferation, scar minimizing, and regenerating skin to shorten cure time.

Many wound dressings, including a polymer containing human skin cells (fibroblasts and/or keratinocytes), have been commercialized. For instance, Dermagraft, Apligraf, and TransCyte have shown effectiveness in burn treatment. However, these products are costly, require complicated storage methods, and can increase the risk of immune reactions [5]. Hence, the creation of effective, long-time stored, affordable cost products to apply in wound management in low- and middle-income countries is in great demand.

For the fabrication of wound dressing, a suitable material on effectiveness and cost should be considered. There are various materials to make wound dressings, such as hydrogels, sodium alginate, collagen, foams, and hydrocolloids [6-10]. Hydrogels are considered the best materials in generating wound dressings because there are dominant features such as hydrophilic nature, soft tissue-like properties, a cooling and soothing effect on the skin [8,11-13]. In this study, human amniotic membrane hydrogel (amnion hydrogel) was used to create wound dressing. An amnion is a membrane on the inner side of the fetal placenta. Amnion consists of 3 layers: A thin epithelial layer, a thick basement membrane of extracellular matrix (ECM) proteins, and an avascular stroma. There are three layers of an avascular stroma: Compact, fibroblast, and intermediate (spongy) layers. Like the human skin, the amnion is composed of extracellular matrix components such as collagen I, III, IV, V, VI, fibronectin, laminin, which provide stiffness and elasticity for amnion [2,14,15]. Amnion has been demonstrated for its anti- 
inflammatory characteristic, anti-bacterial and anti-viral features [2]. Based on these mentioned properties, the amnion can be applied to create products for wound healing purposes [5]. However, using the intact amnion to cover the wound directly has some difficulties in routine use. Limitations related to the difficulty of handling the thin membrane over the injury without folding or tearing, fixing in place on the wound requires the support of sutures or adhesives [16]. Besides, the transportation and storage of the living tissue are also complicated for routine clinical use [16-19]. In 2017, Murphy et al. [20] developed a new product for wound treatment from amnion-containing cell-free solution. That hydrogel was created by combining the solubilized amniotic membrane with hyaluronic acid. Its efficiency was proved in a mouse wound model. In 2020, another work of Murphy et al. [16] showed the wound-healing efficacy of amnion hydrogel and lyophilized amnion membrane powder in a full-thickness porcine skin wound model. These studies demonstrated that amnion hydrogel treatment contributes to improving wound healing. Nevertheless, the degradation of hydrogel could become much faster in aqueous environments such as body fluids [19]. Hence, to overcome these limitations and apply the advantages of the amnion, this study aims to create sponges from human amnion hydrogel using crosslinking and freeze-drying techniques for application as a wound dressing.

\section{Materials and methods}

\section{Ethics statement}

This study was approved by the Medical Ethics Committee of Hung Vuong Hospital, Ho Chi Minh City, Vietnam (the decision number of the permission from Ethical committee: 2395/GCN-BVHV, Hung Vuong Hospital, Ho Chi Minh City, Vietnam). The human amnion was obtained at Hung Vuong Hospital and were accepted by the donors.

\section{The fabrication of sponges}

The human amnion was decellularized, handled to make pre-hydrogel solution, and provided by the Laboratory of Tissue Engineering and Biomedical Materials, University of Science, Vietnam National University, Ho Chi Minh City (Vietnam). The amnion pre-hydrogel structure was white fluid gel. The hydrogel was formed by incubating the pre-hydrogel at $37^{\circ} \mathrm{C}$ for $1 \mathrm{~h}$. The hydrogel was then crosslinked by immersing in $0.625 \%$ glutaraldehyde (GA, Merck, Germany) at $4{ }^{\circ} \mathrm{C}$ for $24 \mathrm{~h}$. There are 3 ways to remove the residual GA: (1) rinse the samples with $1 \mathrm{X}$ phosphate-buffered saline (PBS, Gibco, USA) for 3 days, (2) wash with $1 \%$ glycine solution (Himedia, India) for 1 day and rinse with 1 X PBS for 2 days, and (3) wash with $1 \%$ glycine for 2 days and rinse with $1 \mathrm{X}$ PBS for 1 day. After washing, the samples were kept at $-86{ }^{\circ} \mathrm{C}$ for $24 \mathrm{~h}$ and underwent a drying process (BenchTop Pro 3L XL-75, SP Scientific, USA). The sponges were sterilized by gamma irradiation ( $25 \mathrm{kGy})$.

Based on the 3 removed ways for the residual GA, 3 types of sponges were created: MGA-0 (only washed with PBS), MGA-1 (washed in glycine $1 \%$ for 1 day), MGA-2 (washed in glycine $1 \%$ for 2 days). Moreover, a non-crosslinked sponge (M0) was used as a control.

\section{Investigation of the sponge characterizations}

Structure analysis

The surface of the sponges was observed by the scanning electron microscope (SEM, JSM-6510 JEOL, Japan).

\section{Mechanical test}

To examine the mechanical properties such as compression or expansion of the sponges, the tensile strength formula was used;

Tensile strength $=\mathrm{F} / \mathrm{S}(\mathrm{MPa})$,

where $\mathrm{F}$ is the maximum load that the sponge can withstand $(\mathrm{N})$, and $\mathrm{S}$ is the sample area $\left(\mathrm{mm}^{2}\right)$.

\section{Swelling ratio}

To consider the water-absorbing ability of the sponges, the samples $\left(1.5 \times 2 \mathrm{~cm}^{2}\right)$ were immersed in $3 \mathrm{~mL}$ A solution (sodium chloride $2.298 \mathrm{~g} / \mathrm{L}$, calcium chloride dihydrate $0.368 \mathrm{~g} / \mathrm{L}$, and distilled water). The sponge mass was measured before and after immersing. The swelling ratio was calculated according to the following equation; 
Swelling ratio $(\%)=\frac{W_{e}-W_{0}}{W_{0}} \times 100$,

where $\mathrm{W}_{0}$ is the initial mass of specimens, $\mathrm{W}_{\mathrm{e}}$ is the mass of specimens at the time of 0,24 and $48 \mathrm{~h}$.

\section{In vitro cytotoxicity}

In vitro cytotoxicity was assessed using (3-(4,5-Dimethylthiazol-2-yl)-2,5-diphenyltetrazolium bromide (MTT) method (ISO 10993-5:2009). Experimental groups include negative control (culture medium), positive control (the extraction of medical latex gloves), and sponges MGA-0, MGA-1, MGA2.

A sample was cut into $1.5 \times 2 \mathrm{~cm}^{2}$ pieces. The liquid extract was obtained by incubating the pieces in $0.5 \mathrm{~mL}$ of medium DMEM/F12 (Sigma-Aldrich, USA) supplemented $10 \%$ fetal bovine serum (FBS; Sigma-Aldrich, USA) and $1 \%$ penicillin/streptomycin (Sigma-Aldrich, USA) at $37{ }^{\circ} \mathrm{C}$ for $24 \mathrm{~h}$. Human fibroblasts, which were provided by the Laboratory of Tissue Engineering and Biomedical Materials, were seeded in a 96 well-plate at a cell density of $10^{3}$ cells per well, and incubated at $37{ }^{\circ} \mathrm{C}, 5 \% \mathrm{CO}_{2}$. When the cells reached $80 \%$ of the confluence, they were treated with liquid extract. After $24 \mathrm{~h}$, the liquid extract or medium was discarded; the cells were washed once with $1 \mathrm{X}$ PBS. $0.5 \mathrm{mg} / \mathrm{mL}$ MTT (Sigma-Aldrich, USA) solution was added into each well and incubated for $4 \mathrm{~h}$ at $37{ }^{\circ} \mathrm{C}$. Formazan crystals were dissolved in Dimethylsulfoxide/Ethanol (1:1) (Dimethylsulfoxide, SigmaAldrich, USA; Ethanol, Merck, Germany). The number of active cells was estimated by measuring absorbance at $570 \mathrm{~nm}$ in the Biochrom EZ Read 400 Microplate Reader (Biochrom, United Kingdom).

\section{Statistical analysis}

Each experiment was performed 3 times. Statistical analyses were performed by 1-way analysis of variance (ANOVA). It has statistically significant when $p$-value $<0.05$.

\section{Results and discussion}

\section{Morphology and structure of the sponges}

The amnion pre-hydrogel was a fluid gel (Figure 1A). It has built up a hydrogel phase after incubating at $37^{\circ} \mathrm{C}$ for $1 \mathrm{~h}$ (Figure 1B). However, the amnion hydrogel was degraded after incubating for $5 \mathrm{~min}$ at room temperature (Figure 1C). Thus, the crosslinked method was used to improve the hydrogel structure at room temperature.
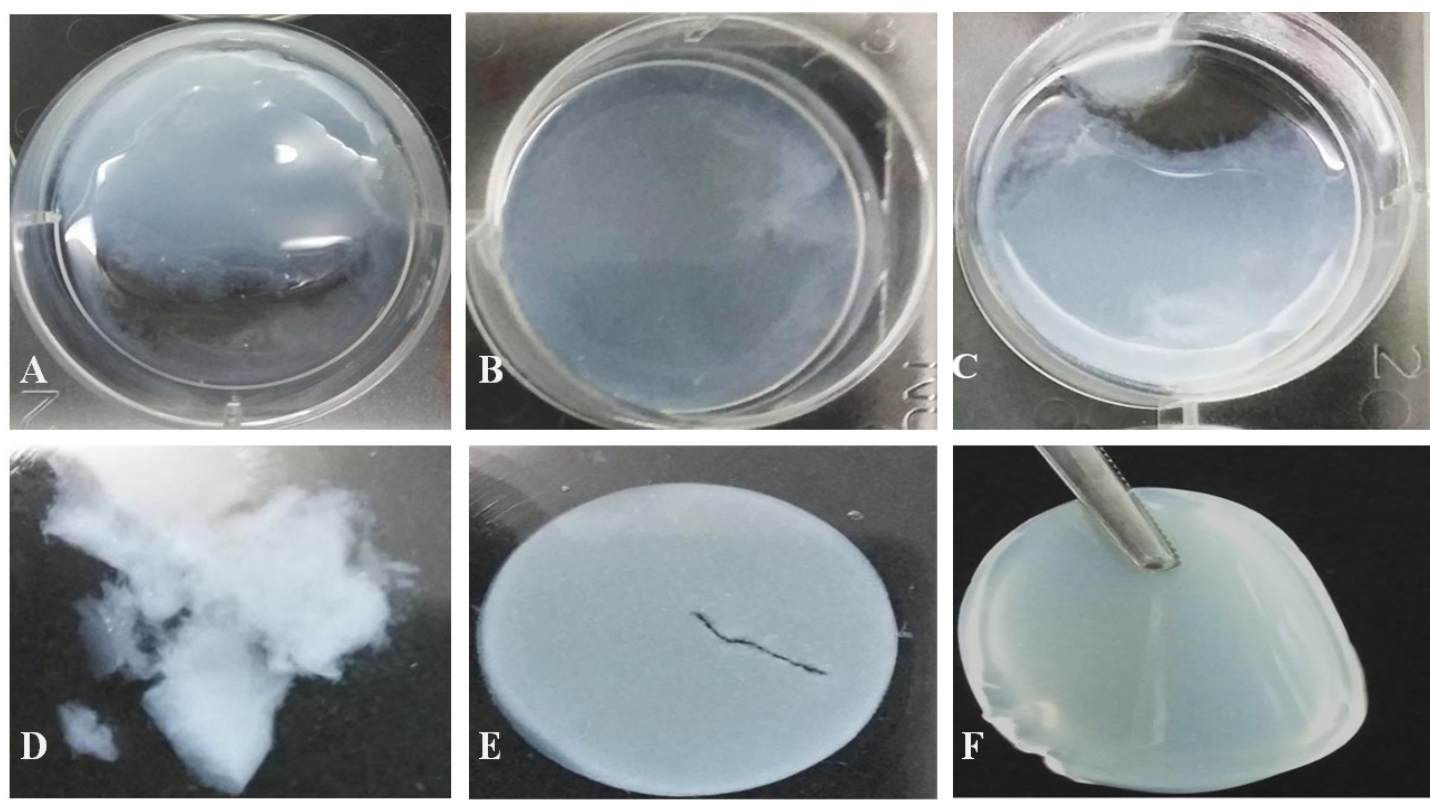

Figure 1 Amnion hydrogel before and after crosslinking; A) pre-hydrogel, B) pre-hydrogel after incubating at $37^{\circ} \mathrm{C}$ for $1 \mathrm{~h}$ (hydrogel), C) hydrogel at room temperature after $5 \mathrm{~min}$, D) hydrogel at room temperature after $24 \mathrm{~h}$, E) and F) GA crosslinked hydrogel after $24 \mathrm{~h}$. 
The results showed the crosslinked samples could preserve the shape when immersed in distilled water (Figure 1E) or pick up out of a container (Figure 1F). After freeze-drying, there were 4 types of sponges: Non-crosslinked sponge (M0), GA crosslinked sponges (MGA: MGA-0, MGA-1, MGA-2) (Figure 2).
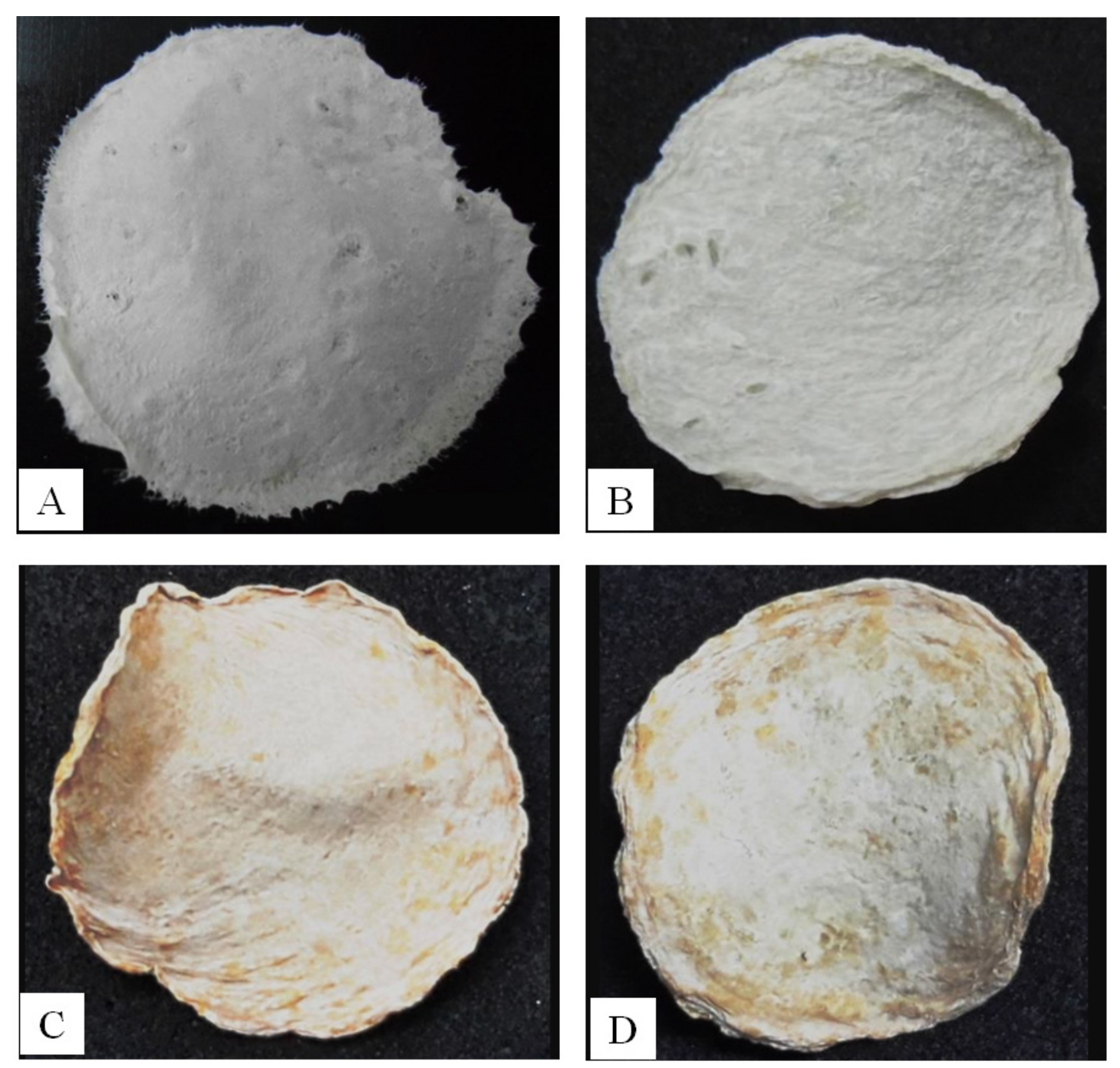

Figure 2 Sponges from amnion hydrogel after freeze-drying; A) M0, B) MGA-0, C) MGA-1 and D) MGA-2.

The M0 and MGA-0 sponge colors were white (Figures 2A and 2B), while the MGA-1 and MGA-2 were yellowish (Figures 2C and 2D). The borders of MGA-0, MGA-1, and MGA-2 were smooth and one was serrations in the border of the M0. A scanning electron microscopy was used to compare the surface structure of the sponges. SEM images displayed a generally flat surface texture of M0 and MGA sponges. A fibrous-like structure can be observed in M0, which become more visible in MGA. Some areas with visible fibrous structure can be detected on MGA surface (Figure 3 B white arrows). 

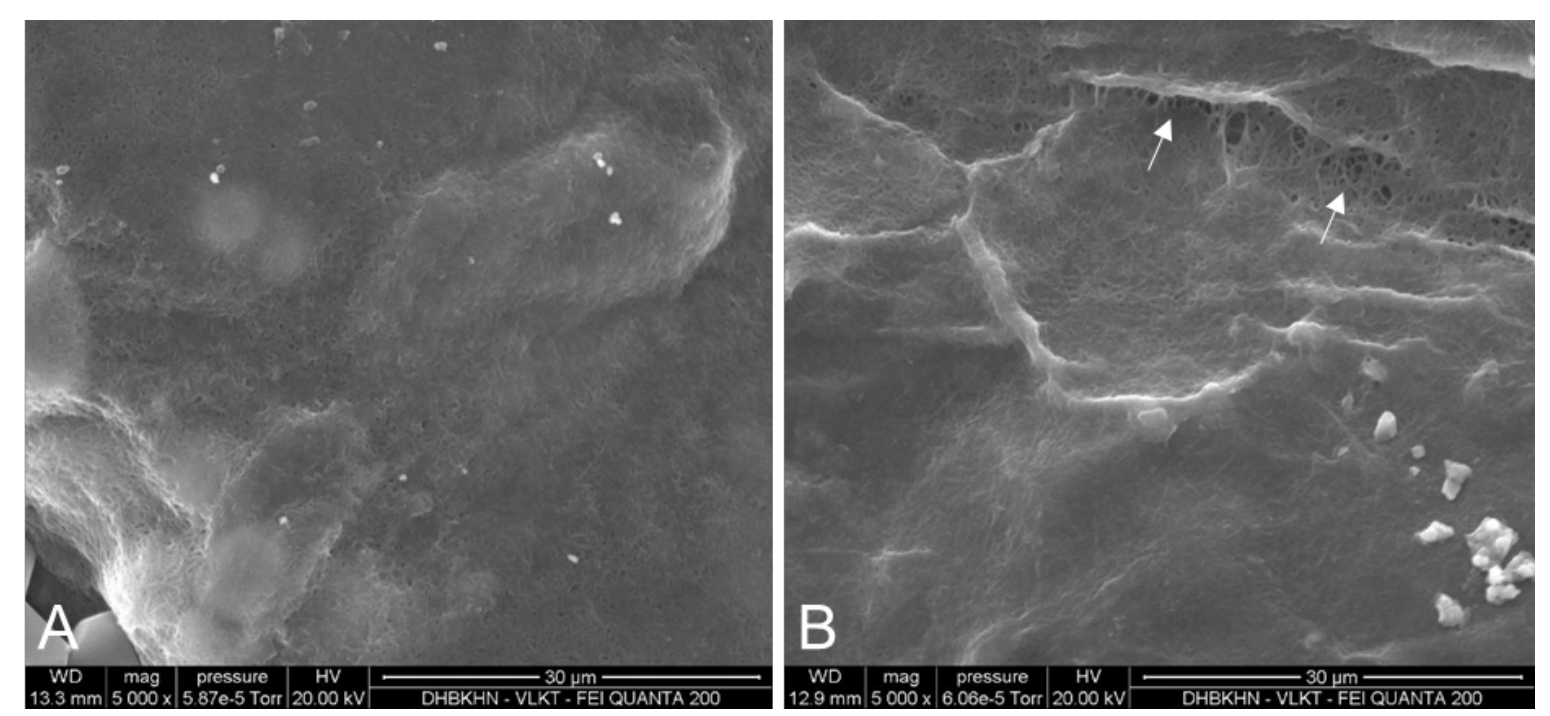

Figure 3 Surface structure of the different sponges; A) M0 sponge and B) MGA sponge.

\section{Mechanical test}

The M0 sponge could be ignored because it broke easily during the test processing. The tensile results of the MGA-0, MGA-1 and MGA-2 were $1.87 \pm 0.37,2.38 \pm 0.25$ and $1.85 \pm 0.28$, respectively (Figure 4). The tensile strength of the MGA-1 was the largest in three sponges, and it is statistically significant compared to the MGA-0 and the MGA-2 $(p<0.05)$. For the MGA-0 and the MGA-2, the tensile difference is nonsignificant $(p>0.05)$.

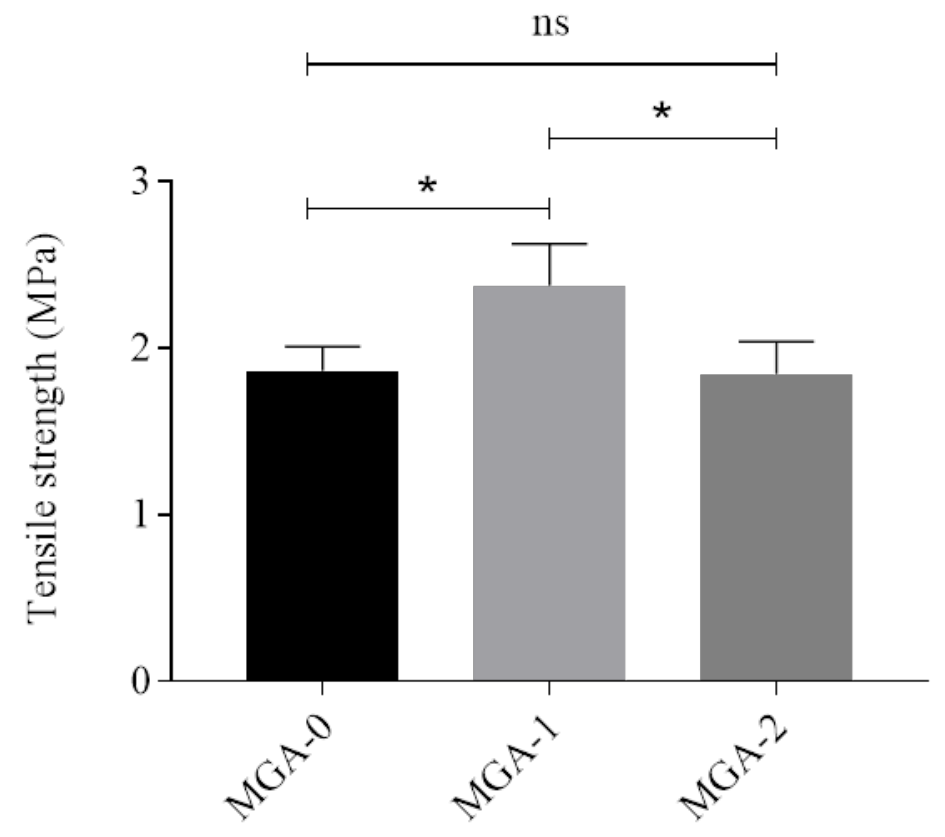

Figure 4 The tensile strength of the MGA-0, MGA-1 and MGA-2 sponges; ns: Not significant, *: $p$-value $<0.05$. 


\section{Swelling ratio}

The results of the swelling ratio of the MGA-0, MGA-1 and MGA-2 were shown in Figures 5 and 6. The M0 sponge was not investigated because it was disintegrated after immersing in A solution in Figure 5.
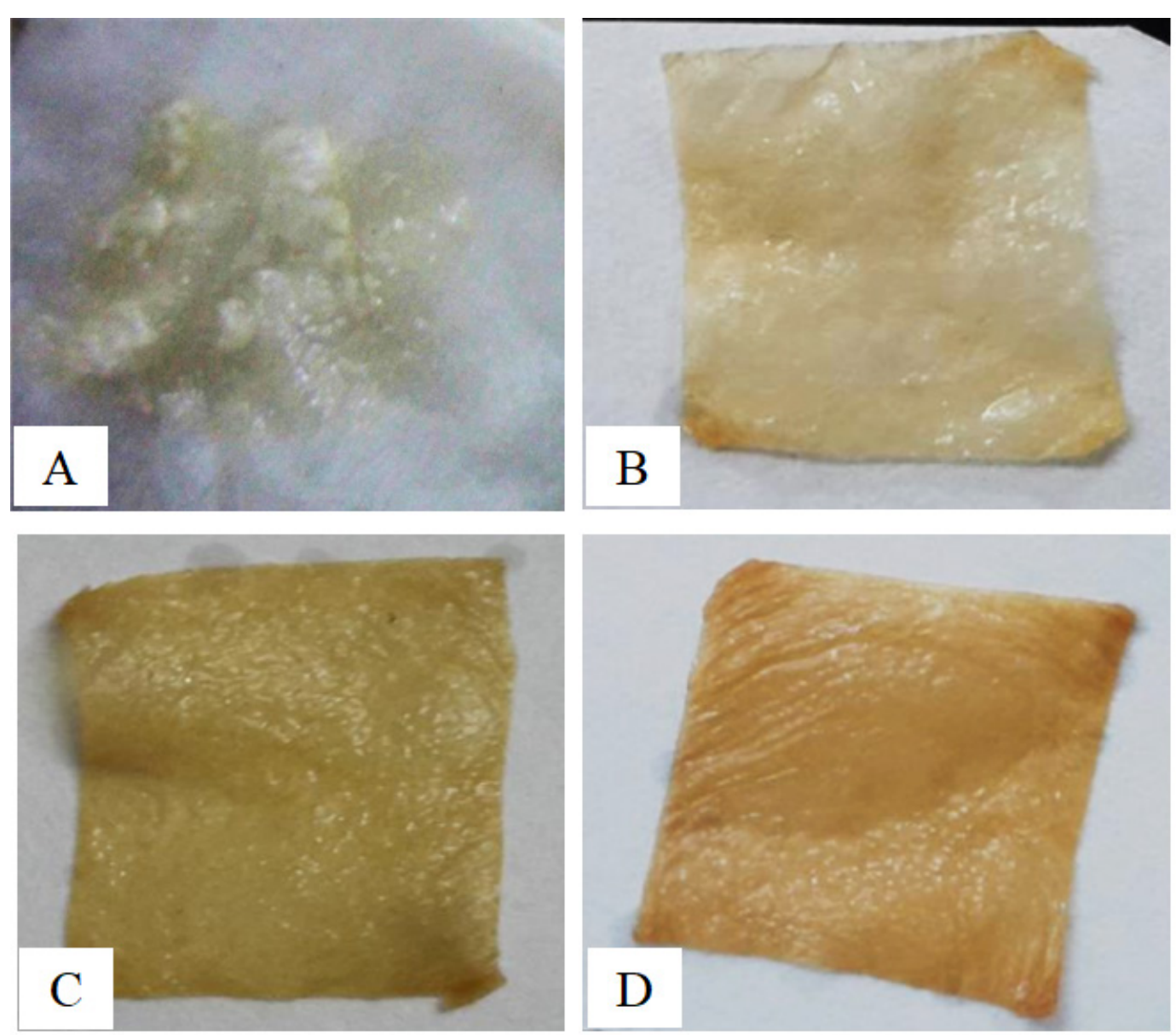

Figure 5 Snapshot of the M0, MGA-0, MGA-1 and MGA-2 sponges after immersing A solution; A) M0, B) MGA-0, C) MGA-1 and D) MGA-2.

The swelling ratio of the MGA-0, MGA-1 and MGA-2 had an increasing trend when the measured time was from 0 to $24 \mathrm{~h}$. From 24 to $48 \mathrm{~h}$, the swelling of MGA-0 increased linearly; however, for the MGA-1 and MGA-2, the obtained results were unchanged. The swelling ratio of the MGA-0, MGA-1 and MGA-2 had statistical significance between the observed time at 0 and $24 \mathrm{~h}$, but they were nonsignificant between 24 and $48 \mathrm{~h}$. At $48 \mathrm{~h}$. The MGA-0 had the largest swelling, and there was a statistical significance comparing to the MGA-1 and MGA-2. However, there was nonsignificant MGA-1 comparing to MGA-2, as shown in Figure 6. 


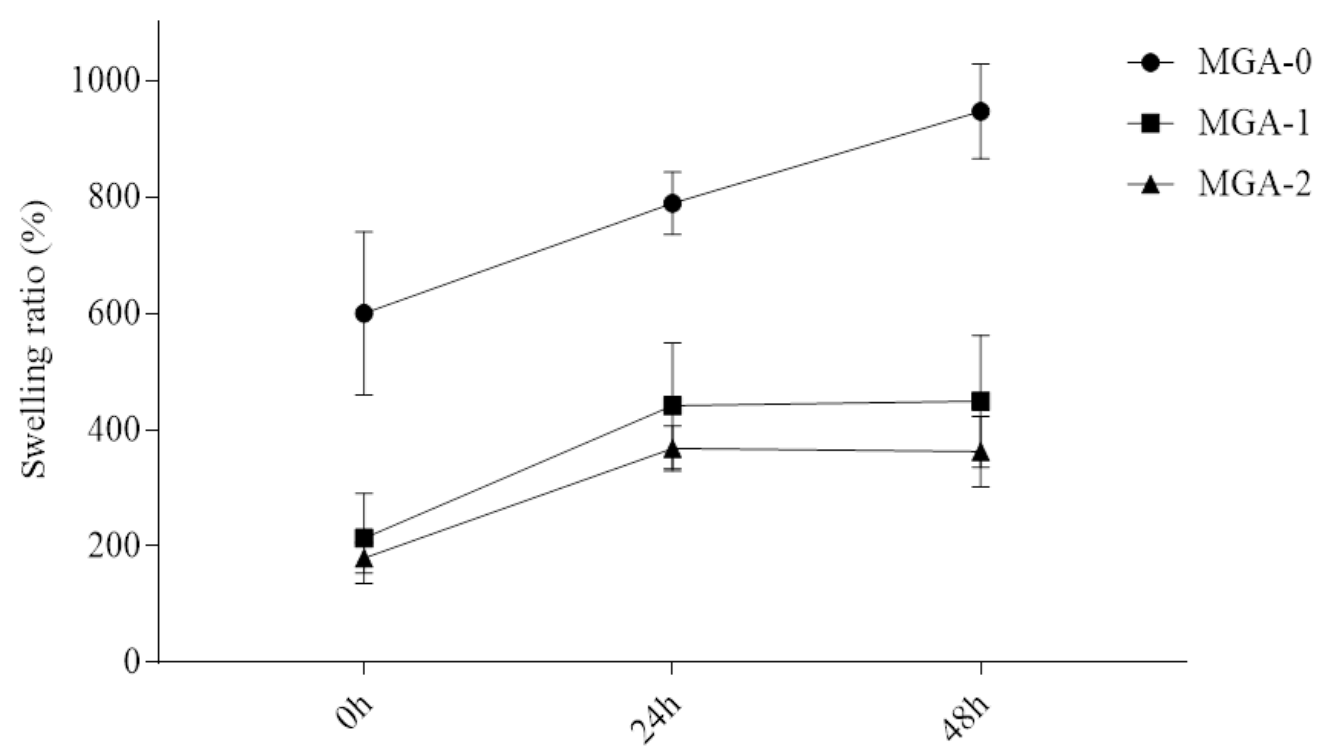

Figure 6 The swelling ratio of the sponges at different time points.

\section{In vitro cytotoxicity}

The results showed that the sponges have cytotoxicity (Figure 7). The relative growth rates (RGR, \%) of the MGA-0, MGA-1, MGA-2 sponges are lower than $70 \%$. Furthermore, it is not significant in comparison with the positive control (the extraction of medical latex gloves). Maybe, the reason for these sponges' cytotoxicity is the residual glutaraldehyde. To remove this residue glutaraldehyde, all sponges were washed by 1 X PBS within 1 day and re-obtain the extract and again the progress.

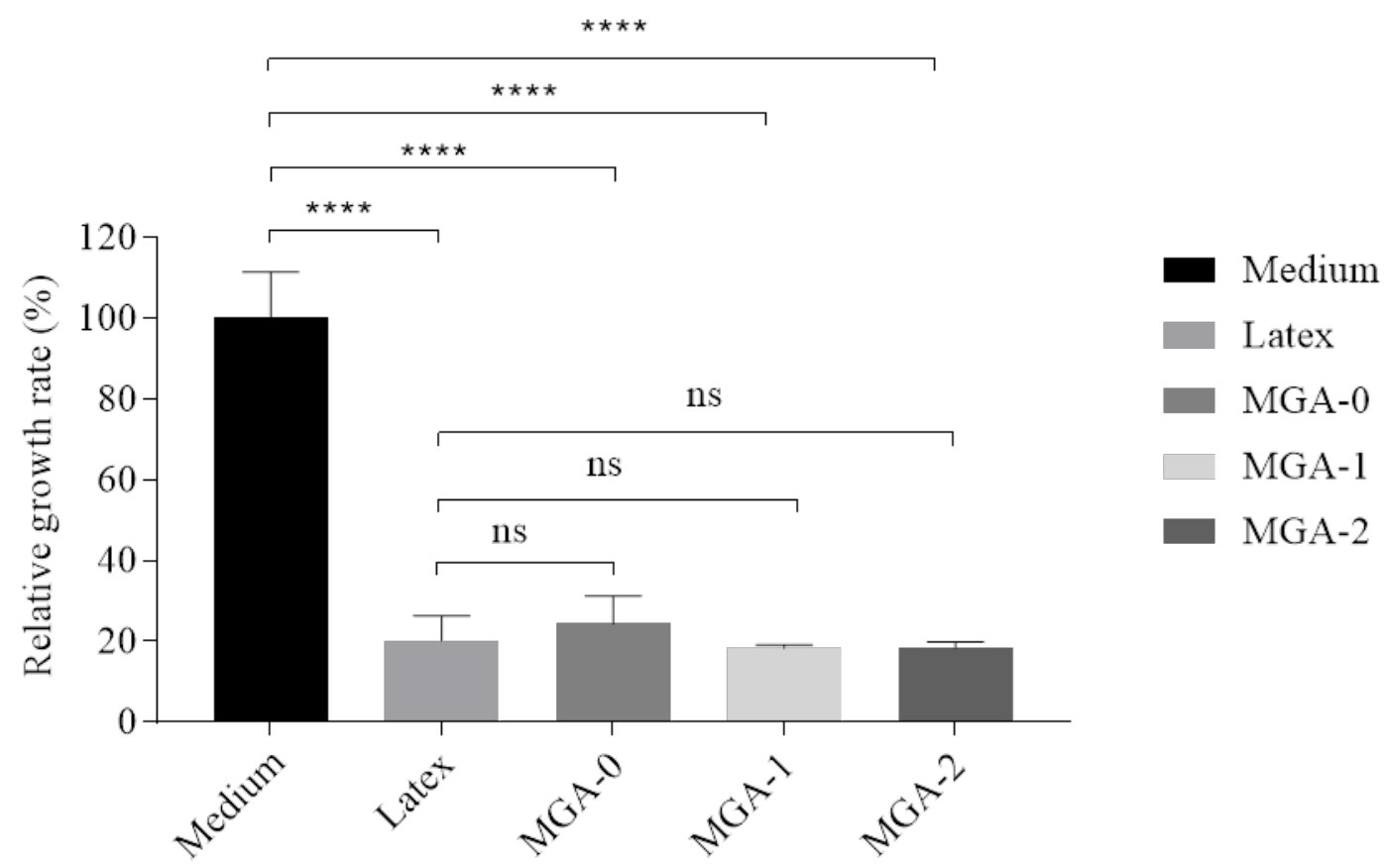

Figure 7 Relative growth rate (\%RGR) of fibroblast of the MGA-0, MGA-1, MGA-2 sponges, and Latex and medium after $24 \mathrm{~h}$ by MTT assay; ns: Not significant, $* * * *$ : $p$-value $<0.0001$. 
In vitro cytotoxicity of all sponges was considered after washing by the $1 \mathrm{X}$ PBS (Figure 8). The results showed that the relative growth rates are increasing. However, only MGA- $0, \%$ RGR is higher than $70 \%$, but for MGA-1 and MGA-2, one is still lower.

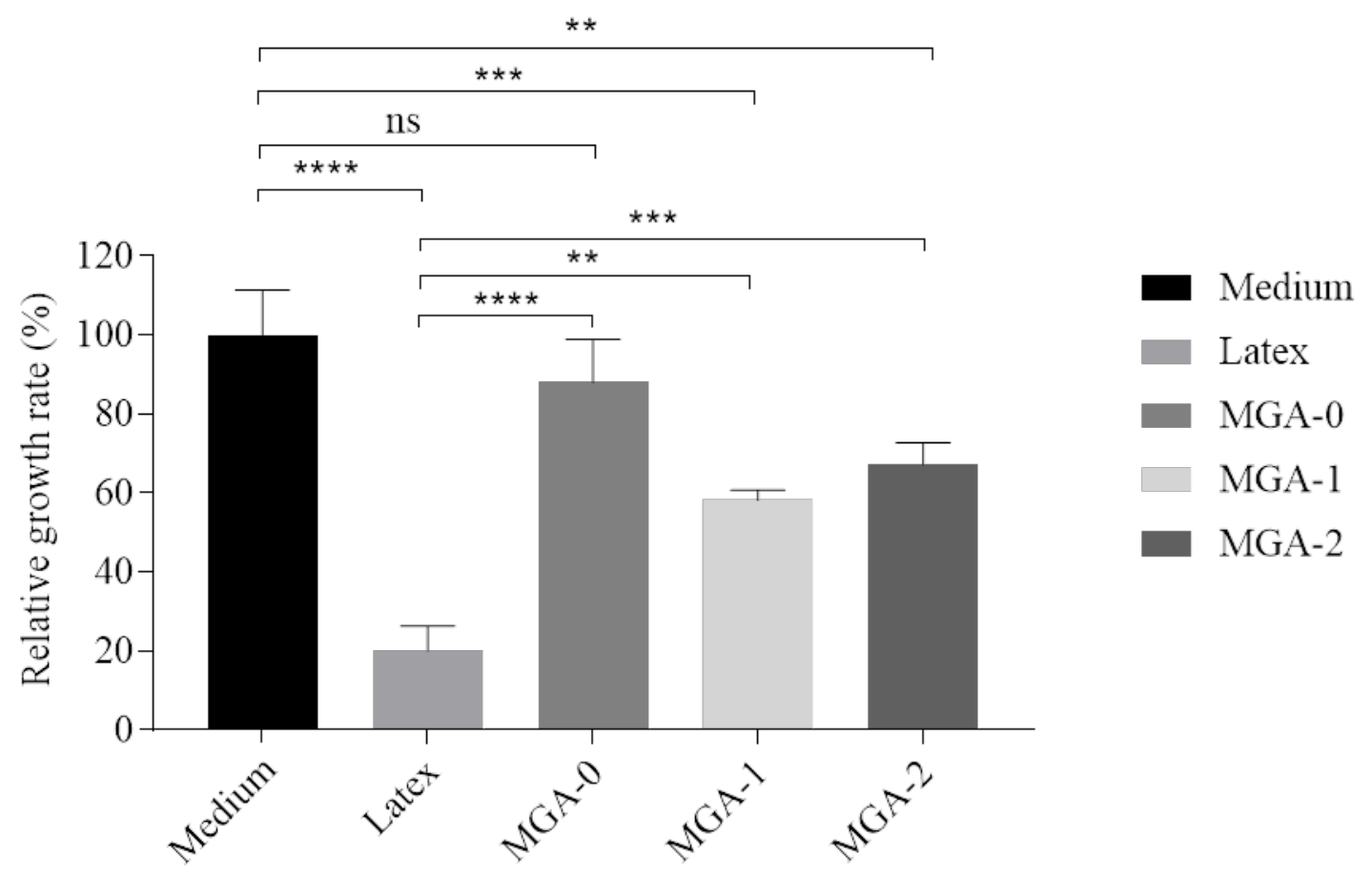

Figure 8 Relative growth rate of fibroblasts of the sponges, latex and medium after washing by $1 \mathrm{X}$ PBS; ns: Not significant, $* *$ : p-value $<0.01, * * *$ : p-value $<0.001: * * * *$ : p-value $<0.0001$.

\section{Discussion}

This work generated wound dressing with the sponge structure by the freeze-drying method. Noncrosslinked sponges are easy to break; hence, to improve structure stabilization, $0.625 \%$ GA was applied as the crosslinker because it is the common chemical crosslinker and has not been disrupted by temperature [21,22]. The GA has 2 aldehyde groups in the structure; this will enhance the ability to crosslink. In addition, with a small size, it is easy to penetrate the sponge structure. The aldehyde groups of GA react with the amine groups of protein to form stable crosslinks [23,24]. The obtained results showed that the crosslinked hydrogel was more difficult to disintegrate. However, the residue GA can induce cytotoxicity [25], so it needs to be removed in the sponges. To remove residual GA, we washed the samples in 1X PBS solution or 1X PBS solution containing glycine. The \%RGR of 3 sponges showed that they were cytotoxic (Figure 7). These results could be predicted that the sponges had residual GA; hence the sponges were again washed by $1 \mathrm{X}$ PBS for $24 \mathrm{~h}$. As expected, the \%RGR of these sponges was improved (Figure 8), and we obtained a non-cytotoxic sponge (MGA-0). Nevertheless, the MGA-1 and MGA-2 were still cytotoxic. This can be explained that the residual GA in the MGA-0 was almost eliminated, while the MGA-1 and MGA-2 might not have completely removed. The results showed that removing the residual GA with $1 \mathrm{X}$ PBS was effective compared with Gly.

In addition, some other criteria were also considered, including mechanical test, swelling ratio. For the mechanical test aspect, the aldehyde groups of GA react with the amine groups of protein to form a stable crosslink, enhancing the stiffness in the crosslinked sponges. The tensile strength of the obtained sponges is about $1.8 \mathrm{MPa}$, and it is similar to the tensile strength of human skin [25]. For the swelling ratio, the sponges can absorb much water quickly. Due to the sponges being fabricated by the freezedrying method, the water in the structure was removed. In the work of Ngadaonye et al. [23], the swelling ratio was about $250-400 \%$ after $24 \mathrm{~h}$. In our study, the swelling ratio of MGA-0, MGA-1, MGA-2 was $790 \pm 54,441 \pm 108$ and $368 \pm 40 \%$, respectively. These results showed that the swelling ratio was 
suitable as supported by the study of Ngadaonye et al. [23]. In general, our work created the sponges from human amnion hydrogel by crosslinking and freeze-drying techniques. This study contributes to the use of extracellular matrix-based biomaterials for wound healing.

\section{Conclusions}

In this study, the MGA-0, MGA-1 and MGA-2 sponges were created by combining freeze-drying with $0.625 \%$ glutaraldehyde. The results showed that the crosslinked sponges had the tensile strength and swelling ratio better than the uncrosslinked sponge (M0). In general, these sponges are cytotoxic. However, after washing again with $1 \mathrm{X}$ PSB, the MGA-0 sponge was non-cytotoxic. The obtained results suggested that the MGA- 0 sponge is a potential candidate in wound dressing application.

\section{Acknowledgments}

This research is funded by University of Science, VNU-HCM under grant number T2020-15.

\section{References}

[1] J Alipour, Y Mehdipour and A Karimi. Epidemiology and outcome analysis of 3030 burn patients with an ICD-10 approach. Ann. Burns Fire Disasters 2020; 33, 3-13.

[2] C Malhotra and AK Jain. Human amniotic membrane transplantation: Different modalities of its use in ophthalmology. World J. Transplant. 2014; 4, 111-21.

[3] K Dhaliwal and N Lopez. Hydrogel dressings and their application in burn wound care. Br. J. Community Nurs. 2018; 23, S24-S27.

[4] A Clement and G Pins. Engineering the tissue-wound interface: Harnessing topography to direct wound healing. Wound Healing Biomater. 2016; 1, 253-75.

[5] SV Murphy, A Skardal, RAJ Nelson, K Sunnon, T Reid, C Clouse, ND Kock, J Jackson, S Soker and A Atala. Amnion membrane hydrogel and amnion membrane powder accelerate wound healing in a full thickness porcine skin wound model. Stem Cells Transl. Med. 2020; 9, 80-92.

[6] M Tenehaus and HO Rennekampff. Topical agents and dressings for local burn wound care. UpToDate, 2018.

[7] S Sharma, A Dua and A Malik. Third generation materials for wound dressings. Int. J. Pharmaceut. Sci. Res. 2014; 5, 2113-24.

[8] EA Kamoun, ERS Kenawy and X Chen. A review on polymeric hydrogel membranes for wound dressing applications: PVA-based hydrogel dressings. J. Adv. Res. 2017; 8, 217-33.

[9] D Queena, JH Evansa, JDS Gaylora, JM Courtneya and WH Reid. Burn wound dressings - a review. Burns 1987; 13, 218-28.

[10] S Dhivya, VV Padma and E Santhini. Wound dressings - a review. BioMedicine (Taipei) 2015; 5, 22.

[11] M Madaghiele, C Demitri, A Sannino and L Ambrosio. Polymeric hydrogels for burn wound care: Advanced skin wound dressings and regenerative templates. Burns Trauma 2014; 2, 153-61.

[12] $\mathrm{C}$ Weller and V Team. Interactive dressings and their role in moist wound management. In: S Rajendran (Eds.). Advanced textiles for wound care. $2^{\text {nd }}$ ed. Woodhead Publishing Limited, Sawston, 2019, p. 105-34.

[13] S Tavakoli and AS Klar. Advanced hydrogels as wound dressings. Biomolecules 2020; 10, 1169.

[14] AC Mamede, MJ Carvalho, AM Abrantes, M Laranjo, CJ Maia and MF Botelho. Amniotic membrane: From structure and functions to clinical applications. Cell Tissue Res. 2012; 349, 44758.

[15] S Parry and JF Strauss $3^{\text {rd }}$. Premature rupture of the fetal membranes. N. Engl. J. Med. 1998; 338, 663-70.

[16] SV Murphy, A Skardal, RAJ Nelson, K Sunnon, T Reid, C Clouse, ND Kock, J Jackson and S Soker. A Atala. Amnion membrane hydrogel and amnion membrane powder accelerate wound healing in a full thickness porcine skin wound model. Stem cells Transl. Med. 2020; 9, 80-92.

[17] DE Fetterolf and RJ Snyder. Scientific and clinical support for the use of dehydrated amniotic membrane in wound management. Wounds 2012; 24, 299-307.

[18] J Oba, M Okabe, T Yoshida, C Soko, M Fathy, K Amano, D Kobashi, M Wakasugi and H Okudera. Hyperdry human amniotic membrane application as a wound dressing for a full-thickness skin excision after a third-degree burn injury. Burns Trauma 2020; 8, 014. 
[19] S Leal-Marin, T Kern, N Hofmann, O Pogozhykh, C Framme, M Börgel, C Figueiredo, B Glasmacher and $\mathrm{O}$ Gryshkov. Human amniotic membrane: A review on tissue engineering, application, and storage. J. Biomed. Mater. Res. B Appl. Biomater. 2020; 109, 1198-215.

[20] SV Murphy, A Skardal, L Song, K Sutton, R Haug, DL Mack, J Jackson, S Soker and A Atala. Solubilized amnion membrane hyaluronic acid hydrogel accelerates full-thickness wound healing. Stem Cells Transl. Med. 2017; 6, 2020-32.

[21] Q Yao, YW Zheng, HL Lin, QH Lan, ZW Huang, LF Wang, R Chen, J Xiao, L Kou, HL Xu and YZ Zhao. Exploiting crosslinked decellularized matrix to achieve uterus regeneration and construction. Artif. Cell. Nanomed. Biotechnol. 2020; 48, 218-29.

[22] MT Wolf, KA Daly, EP Brennan-Pierce, SA Johnson, CA Carruthers, A D'Amore, SP Nagarkar, SS Velankar and SF Badylak. A hydrogel derived from decellularized dermal extracellular matrix. Biomaterials 2012; 33, 7028-38.

[23] I Migneault, C Dartiguenave, MJ Bertrand and KC Waldron. Glutaraldehyde: Behavior in aqueous solution, reaction with proteins, and application to enzyme crosslinking. Biotechniques 2004; 37, 790-802.

[24] LHHO Damink, PJ Dijkstra, MJAV Luyn, PBV Wachem, P Nieuwenhuis and J Feijen. Glutaraldehyde as a crosslinking agent for collagen-based biomaterials. J. Mater. Sci. Mater. Med. $1995 ; 6,460-72$.

[25] LM Delgado, K Fuller and DI Zeugolis. Collagen cross-linking: Biophysical, biochemical, and biological response analysis. Tissue Eng. A 2017; 23, 1064-77. 